\title{
A review on phenolic resin and its composites
}

\begin{abstract}
Background: Phenolic resins received greater attention from academic researchers and made prestigious status among all thermoset resins. Several effective attempts have made to explore different synthesis processes of producing phenolic in more useful and advanced way. Since the date of innovation, huge varieties of biocomposites and composites products have been produced for various applications.
\end{abstract}

Methods: Among all types of Phenolic resin related applications, Phenolic resin molding compounds have been used for highest utility products due to better mechanical strength, resistance, long-term reliability and cost effectiveness.

Results: Currently, modified phenolic resins are broadly used in the fabrication of synthetic/natural fiber reinforced composites in order to replace metals and ceramics on industrial level owing to its superior mechanical, thermal and electrical properties. This review article is intended to present an overview on the phenolic chemistry, curing process, structure, synthesis and its properties.

Conclusion: The present review article also aims to cover the reported research works on modified phenolic, and bio-phenolic resins, and their diverse industrial applications to support the ongoing research and development in green sustainable commercial products.

Keyword: Phenolic resin; Natural fibres; Phenolic curing process; Sustainable phenolic; Composite; Hybrid composite; Fire retardant 